Document downloaded from:

http://hdl.handle.net/10251/107404

This paper must be cited as:

Boiti, C.; Jornet Casanova, D. (2017). A simple proof of Kotake-Narasimhan theorem in some classes of ultradifferentiable functions. Journal of Pseudo-Differential Operators and Applications. 8(2):297-317. doi:10.1007/s11868-016-0163-y

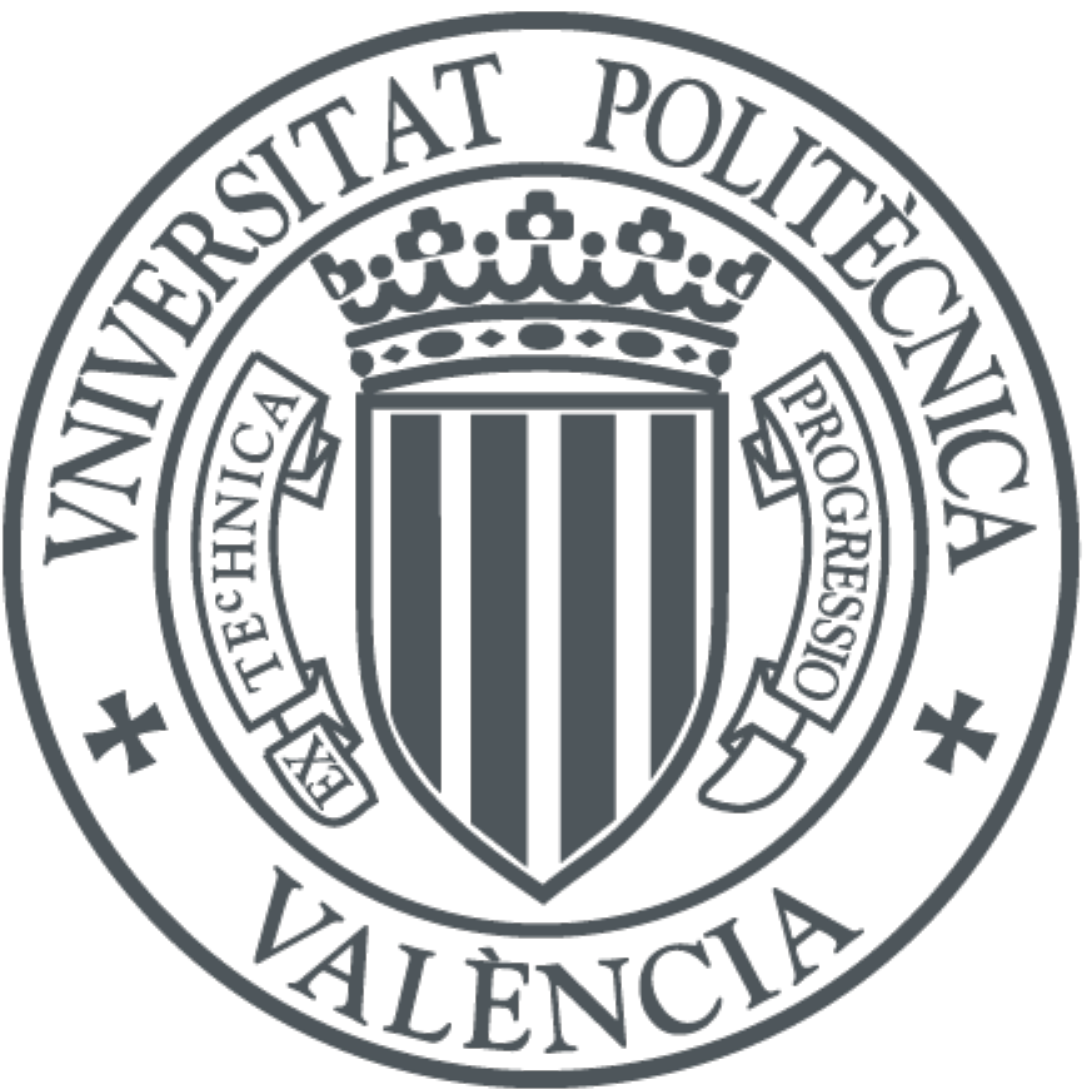

The final publication is available at

http://doi.org/10.1007/s11868-016-0163-y

Copyright Springer

Additional Information 


\title{
A simple proof of Kotake-Narasimhan theorem in some classes of ultradifferentiable functions
}

\author{
Chiara Boiti · David Jornet
}

Received: date / Accepted: date

\begin{abstract}
We give a simple proof of a general theorem of Kotake-Narasimhan for elliptic operators in the setting of ultradifferentiable functions in the sense of Braun, Meise and Taylor. We follow the ideas of Komatsu. Based on an example of Metivier, we also show that the ellipticity is a necessary condition for the theorem to be true.
\end{abstract}

Keywords Iterates of an operator, Theorem of Kotake-Narasimhan, ultradifferentiable functions.

Mathematics Subject Classification (2000) 46E10 - 46F05

\section{Introduction and main result}

The problem of iterates began when Komatsu [13] in 1960 characterized analytic functions $f$ in terms of the behaviour of successive iterates $P(D)^{j} f$ of the function $f$ for a linear partial differential elliptic operator $P(D)$ with constant coefficients. He proved that a $C^{\infty}$ function $f$ is real analytic in $\Omega$ if and only

The authors were partially supported by the INdAM-GNAMPA Projects 2014 and 2015. The second author was partially supported by MINECO, Project MTM2013-43540-P.

C. Boiti

Dipartimento di Matematica e Informatica, Università di Ferrara, Via Machiavelli n. 30, I-44121 Ferrara, Italy

Tel.: +390532974018

Fax: +390532974003

E-mail: chiara.boiti@unife.it

D. Jornet

Instituto Universitario de Matemática Pura y Aplicada IUMPA, Universitat Politècnica de València, Camino de Vera, s/n, E-46071 Valencia, Spain

Tel.: +3496387 $7000 \times 88396$

Fax: +34 963879494

E-mail: djornet@mat.upv.es 
if for every compact set $K \subset \subset \Omega$ there is a constant $C>0$ such that

$$
\left\|P(D)^{j} u\right\|_{L^{2}(K)} \leq C^{j+1}(j !)^{m}, \quad \forall j \in \mathbb{N}_{0}:=\mathbb{N} \cup\{0\},
$$

where $m$ is the order of the operator and $\|\cdot\|_{L^{2}(K)}$ is the $L^{2}$ norm on $K$. This result was generalized to the case of elliptic linear partial differential operators $P(x, D)$ with real analytic coefficients in $\Omega$ by Kotake and Narasimhan [16], and is known as "the Theorem of Kotake-Narasimhan". Komatsu [15] gave a simpler proof. Similar results have been previously considered by Nelson [24]. Later these results were extended to Gevrey functions by Newberger and Zielezny [25] in the case of operators with constant coefficients. Lions and Magenes [22] considered the case of Denjoy-Carleman classes of Roumieu type for elliptic linear partial differential operators $P(x, D)$ with variable coefficients in the same Roumieu class, and Oldrich [26] treated the case of Denjoy-Carleman classes of Beurling type with some loss of regularity with respect to the coefficients. Métivier [23] proved that the result of Lions and Magenes for Gevrey classes is true only for elliptic operators in the case of real analytic coefficients. Spaces of Gevrey type given by the iterates of a differential operator are called generalized Gevrey classes and were used by Langenbruch [18-21] for different purposes.

More recently, Juan-Huguet [11] extended the results of Komatsu [13], Newberger and Zielezny [25] and Métivier [23] to the setting of non-quasianalytic classes in the sense of Braun, Meise and Taylor [8] for operators with constant coefficients. In [11], Juan-Huguet introduced the generalized spaces of ultradifferentiable functions $\mathcal{E}_{*}^{P}(\Omega)$ on an open subset $\Omega$ of $\mathbb{R}^{n}$ for a fixed linear partial differential operator $P$ with constant coefficients, and proved that these spaces are complete if and only if $P$ is hypoelliptic. Moreover, Juan-Huguet showed that, in this case, the spaces are nuclear. Later, the same author in [12] established a Paley-Wiener theorem for the classes $\mathcal{E}_{*}^{P}(\Omega)$, again under the hypothesis of the hypoellipticity of $P$.

We used in [3] and [2] the results of Juan Huguet to define and characterize a wave front set for the generalized spaces of ultradifferentiable functions $\mathcal{E}_{*}^{P}(\Omega)$ when $P$ is hypoelliptic. In particular, for $P$ elliptic we obtain a microlocal version of the theorem of Kotake and Narasimhan. In order to remove the assumption on the hypoellipticity of the operator, we considered in [1] a different setting of ultradifferentiable functions, following the ideas of [5].

Here, we give a simple proof of the theorem of Kotake-Narasimhan [16, Theorem 1] in the setting of ultradifferentiable functions as introduced by Braun, Meise and Taylor [8] for quasianalytic or non-quasianalytic weight functions. We will consider subadditive weight functions, or more generally, weight functions which satisfy condition $\left(\alpha_{0}\right)$, that we define later (see for example Petzsche and Vogt [27, p. 19] or Fernández and Galbis [9, p. 401]). We follow the lines of Komatsu [15].

Let us recall from [8] the definitions of weight functions $\omega$ and of the spaces of ultradifferentiable functions of Beurling and Roumieu type:

Definition 11 A non-quasianalytic weight function is a continuous increasing function $\omega:[0,+\infty[\rightarrow[0,+\infty[$ with the following properties: 
( $\alpha) \exists L>0$ s.t. $\omega(2 t) \leq L(\omega(t)+1) \quad \forall t \geq 0$;

( $\beta) \int_{1}^{+\infty} \frac{\omega(t)}{t^{2}} d t<+\infty$,

$(\gamma) \log (t)=o(\omega(t))$ as $t \rightarrow+\infty$;

( $\delta) \varphi_{\omega}: t \mapsto \omega\left(e^{t}\right)$ is convex.

We say that $\omega$ is quasianalytic if, instead of $(\beta)$ it satisfies:

( $\left.\beta^{\prime}\right) \int_{1}^{+\infty} \frac{\omega(t)}{t^{2}} d t=+\infty$.

We will consider also the following property:

$\left(\alpha_{0}\right) \exists C>0, \exists t_{0}>0, \forall \lambda \geq 1, \forall t \geq t_{0}: \omega(\lambda t) \leq \lambda C \omega(t)$.

The property $\left(\alpha_{0}\right)$ above is used in [27, p. 19] and [9, p. 401], for instance. Moreover, a weight function $\omega$ satisfies $\left(\alpha_{0}\right)$ if and only if it is equivalent to a subadditive (or concave) weight function. In the following, we will assume that our weight functions satisfy $\left(\alpha_{0}\right)$, and there is no loss of generality to consider only subadditive weights. This condition should be compared with $[22,(1.4)$, p. 3] or [26, (2), p. 1], which is a similar condition for Denjoy-Carleman classes.

Normally, we will denote $\varphi_{\omega}$ simply by $\varphi$.

For a weight function $\omega$ we define $\bar{\omega}: \mathbb{C}^{n} \rightarrow[0,+\infty[$ by $\bar{\omega}(z):=\omega(|z|)$ and again we denote this function by $\omega$.

The Young conjugate $\varphi^{*}:[0,+\infty[\rightarrow[0,+\infty[$ is defined by

$$
\varphi^{*}(s):=\sup _{t \geq 0}\{s t-\varphi(t)\}
$$

There is no loss of generality to assume that $\omega$ vanishes on $[0,1]$. Then $\varphi^{*}$ has only non-negative values, it is convex, $\varphi^{*}(t) / t$ is increasing and tends to $\infty$ as $t \rightarrow \infty$, and $\varphi^{* *}=\varphi$.

Example 12 The following functions are, after a change in some interval $[0, M]$, examples of weight functions:

(i) $\omega(t)=t^{d}$ for $0<d<1$.

(ii) $\omega(t)=(\log (1+t))^{s}, s>1$.

(iii) $\omega(t)=t(\log (e+t))^{-\beta}, \beta>1$.

(iv) $\omega(t)=\exp \left(\beta(\log (1+t))^{\alpha}\right), 0<\alpha<1$.

In what follows, $\Omega$ denotes an arbitrary subset of $\mathbb{R}^{n}$ and $K \subset \subset \Omega$ means that $K$ is a compact subset in $\Omega$.

Definition 13 Let $\omega$ be a weight function. For a compact subset $K$ in $\mathbb{R}^{n}$ which coincides with the closure of its interior and $\lambda>0$, we define the seminorm

$$
p_{K, \lambda}(f):=\sup _{\alpha \in \mathbb{N}_{0}^{n}} \sup _{x \in K}\left|f^{(\alpha)}(x)\right| \exp \left(-\lambda \varphi^{*}\left(\frac{|\alpha|}{\lambda}\right)\right),
$$

where $\mathbb{N}_{0}:=\mathbb{N} \cup\{0\}$, and set

$$
\mathcal{E}_{\omega}^{\lambda}(K):=\left\{f \in C^{\infty}(K): p_{K, \lambda}(f)<\infty\right\},
$$


which is a Banach space endowed with the $p_{K, \lambda}(\cdot)$-topology.

For an open subset $\Omega$ in $\mathbb{R}^{n}$, the class of $\omega$-ultradifferentiable functions of Beurling type is defined by

$\mathcal{E}_{(\omega)}(\Omega):=\left\{f \in C^{\infty}(\Omega): p_{K, \lambda}(f)<\infty\right.$, for every $K \subset \subset \Omega$ and every $\left.\lambda>0\right\}$.

The topology of this space is

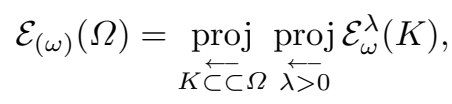

and one can show that $\mathcal{E}_{(\omega)}(\Omega)$ is a Fréchet space.

For an open subset $\Omega$ in $\mathbb{R}^{n}$, the class of $\omega$-ultradifferentiable functions of Roumieu type is defined by:

$$
\mathcal{E}_{\{\omega\}}(\Omega):=\left\{f \in C^{\infty}(\Omega): \forall K \subset \subset \Omega \exists \lambda>0 \text { such that } p_{K, \lambda}(f)<\infty\right\} .
$$

Its topology is the following

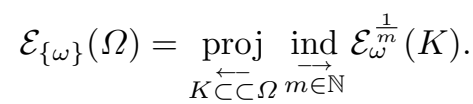

This is a complete PLS-space, that is, a complete space which is a projective limit of LB-spaces. Moreover, $\mathcal{E}_{\{\omega\}}(\Omega)$ is also a nuclear and reflexive locally convex space. In particular, $\mathcal{E}_{\{\omega\}}(\Omega)$ is an ultrabornological (hence barrelled and bornological) space.

The elements of $\mathcal{E}_{(\omega)}(\Omega)$ (resp. $\mathcal{E}_{\{\omega\}}(\Omega)$ ) are called ultradifferentiable functions of Beurling type (resp. Roumieu type) in $\Omega$.

In the case that $\omega(t):=t^{d}(0<d<1)$, the corresponding Roumieu class is the Gevrey class with exponent $1 / d$. In the limit case $d=1$, the corresponding Roumieu class $\mathcal{E}_{\{\omega\}}(\Omega)$ is the space of real analytic functions on $\Omega$ whereas the Beurling class $\mathcal{E}_{(\omega)}\left(\mathbb{R}^{n}\right)$ gives the entire functions. Observe that Gevrey weights satisfy $\left(\alpha_{0}\right)$.

Given a polynomial $P \in \mathbb{C}\left[z_{1}, \ldots, z_{n}\right]$ of degree $m, P(z)=\sum_{|\alpha| \leq m} a_{\alpha} z^{\alpha}$, the partial differential operator $P(D)$ is defined as $P(D)=\sum_{|\alpha| \leq m} a_{\alpha} D^{\alpha}$, where $D=\frac{1}{i} \partial$. Following [11], we consider smooth functions in an open set $\Omega$ such that there exists $C>0$ verifying for each $j \in \mathbb{N}_{0}:=\mathbb{N} \cup\{0\}$,

$$
\left\|P^{j}(D) f\right\|_{L^{2}(K)} \leq C \exp \left(\lambda \varphi^{*}\left(\frac{j m}{\lambda}\right)\right),
$$

where $K$ is a compact subset in $\Omega,\|\cdot\|_{L^{2}(K)}$ denotes the $L^{2}$-norm on $K$ and $P^{j}(D)$ is the $j$-th iterate of the partial differential operator $P(D)$ of order $m$, i.e.,

$$
P^{j}(D)=\underbrace{P(D) \circ \cdots \circ P(D)}_{j} .
$$

If $j=0$, then we set $P^{0}(D) f=f$. 
The spaces of ultradifferentiable functions with respect to the successive iterates of $P$ are defined as follows.

Let $\omega$ be a weight function. Given a polynomial $P$, an open set $\Omega$ of $\mathbb{R}^{n}$, a compact subset $K \subset \subset \Omega$ and $\lambda>0$, we define the seminorm

$$
\|f\|_{K, \lambda}:=\sup _{j \in \mathbb{N}_{0}}\left\|P^{j}(D) f\right\|_{2, K} \exp \left(-\lambda \varphi^{*}\left(\frac{j m}{\lambda}\right)\right)
$$

and set

$$
\mathcal{E}_{P, \omega}^{\lambda}(K)=\left\{f \in C^{\infty}(K):\|f\|_{K, \lambda}<+\infty\right\} .
$$

It is a normed space endowed with the $\|\cdot\|_{K, \lambda}$-norm.

The space of ultradifferentiable functions of Beurling type with respect to the iterates of $P$ is:

$$
\mathcal{E}_{(\omega)}^{P}(\Omega)=\left\{f \in C^{\infty}(\Omega):\|f\|_{K, \lambda}<+\infty \text { for each } K \subset \subset \Omega \text { and } \lambda>0\right\},
$$

endowed with the topology given by

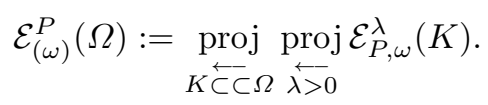

If $\left\{K_{n}\right\}_{n \in \mathbb{N}}$ is a compact exhaustion of $\Omega$ we have

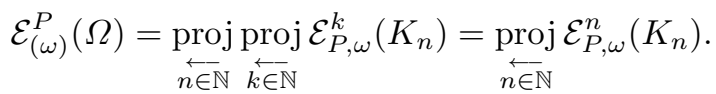

This is a metrizable locally convex topology defined by the fundamental system of seminorms $\left\{\|\cdot\|_{K_{n}, n}\right\}_{n \in \mathbb{N}}$.

The space of ultradifferentiable functions of Roumieu type with respect to the iterates of $P$ is defined by:

$$
\mathcal{E}_{\{\omega\}}^{P}(\Omega)=\left\{f \in C^{\infty}(\Omega): \forall K \subset \subset \Omega \exists \lambda>0 \text { such that }\|f\|_{K, \lambda}<+\infty\right\} .
$$

Its topology is defined by

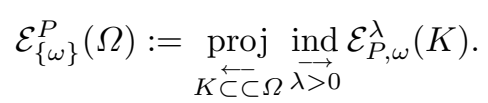

In the following, * will denote either $\{\omega\}$ or $(\omega)$. The inclusion map $\mathcal{E}_{*}(\Omega) \hookrightarrow$ $\mathcal{E}_{*}^{P}(\Omega)$ is continuous (see [11, Theorem 4.1]). The space $\mathcal{E}_{*}^{P}(\Omega)$ is complete if and only if $P$ is hypoelliptic (see [11, Theorem 3.3]). Moreover, under a mild condition on $\omega$ introduced by Bonet, Meise and Melikhov [7, 16 Corollary (3)], $\mathcal{E}_{*}^{P}(\Omega)$ coincides with the class of ultradifferentiable functions $\mathcal{E}_{*}(\Omega)$ if and only if $P$ is elliptic (see [11, Theorem 4.12]).

Now, let $P(x, D)=\sum_{|\alpha|<m} a_{\alpha}(x) D^{\alpha}$ be a linear partial differential operator of order $m$ with smooth coefficients in an open subset $\Omega \subseteq \mathbb{R}^{n}$, i.e. $a_{\alpha} \in C^{\infty}(\Omega)$ for all multi-index $\alpha \in \mathbb{N}_{0}^{n}$ with $|\alpha| \leq m$. We consider the $q$-th 
iterates $P^{q}=P \circ \cdots \circ P$ of $P:=P(x, D)$ and define the corresponding spaces of iterates as above:

$$
\begin{aligned}
\mathcal{E}_{(\omega)}^{P}(\Omega):=\left\{u \in C^{\infty}(\Omega): \quad\right. & \forall K \subset \subset \Omega \forall k \in \mathbb{N} \exists c_{k}>0 \text { s.t. } \\
& \left.\left\|P^{q} u\right\|_{L^{2}(K)} \leq c_{k} e^{k \varphi^{*}(q m / k)} \forall q \in \mathbb{N}_{0}\right\}
\end{aligned}
$$

for the Beurling case, and

$$
\begin{aligned}
\mathcal{E}_{\{\omega\}}^{P}(\Omega):=\left\{u \in C^{\infty}(\Omega): \quad\right. & \forall K \subset \subset \Omega \exists k \in \mathbb{N}, c>0 \text { s.t. } \\
& \left.\left\|P^{q} u\right\|_{L^{2}(K)} \leq c e^{\frac{1}{k} \varphi^{*}(q m k)} \forall q \in \mathbb{N}_{0}\right\}
\end{aligned}
$$

for the Roumieu case.

We generalize some results of Juan-Huguet [11] for operators with variable coefficients in the following way. First, we state our main result in the Roumieu case:

Theorem 14 Let $\omega$ be a subadditive weight function, $\Omega \subseteq \mathbb{R}^{n}$ a domain, i.e. open and connected, and $P(x, D)$ a linear partial differential operator of order $m$ with coefficients in $\mathcal{E}_{\{\omega\}}(\Omega)$. Then:

(i) $\mathcal{E}_{\{\omega\}}(\Omega) \subseteq \mathcal{E}_{\{\omega\}}^{P}(\Omega)$;

(ii) if $P(x, D)$ is elliptic, then $\mathcal{E}_{\{\omega\}}(\Omega)=\mathcal{E}_{\{\omega\}}^{P}(\Omega)$.

In the Beurling case we lose some regularity; compare to Oldrich [26, Teorema 1]:

Theorem 15 Let $\omega$ be a subadditive weight function, $\Omega \subseteq \mathbb{R}^{n}$ a domain and $P(x, D)$ a linear partial differential operator of order $m$ with coefficients in $\mathcal{E}_{(\omega)}(\Omega)$. Then:

(i) $\mathcal{E}_{(\omega)}(\Omega) \subseteq \mathcal{E}_{(\omega)}^{P}(\Omega)$;

(ii) if $P(x, D)$ is elliptic, then $\mathcal{E}_{(\omega)}^{P}(\Omega) \subseteq \mathcal{E}_{(\sigma)}(\Omega)$ for every subadditive weight function $\sigma(t)=o(\omega(t))$ as $t \rightarrow+\infty$.

Theorem 14 is the generalization to the class of ultradifferentiable functions $\mathcal{E}_{\{\omega\}}(\Omega)$ of the theorem of Kotake-Narasimhan for an elliptic linear partial differential operator $P(x, D)$ with coefficients in the same class $\mathcal{E}_{\{\omega\}}(\Omega)$. We observe that the ellipticity of $P$ is not needed for the inclusion $\mathcal{E}_{\{\omega\}}(\Omega) \subseteq$ $\mathcal{E}_{\{\omega\}}^{P}(\Omega)$. However, we show in Example 31 that the ellipticity is necessary for the equality $\mathcal{E}_{\{\omega\}}(\Omega)=\mathcal{E}_{\{\omega\}}^{P}(\Omega)$ for a large family of weights $\omega$. We use the example of Metivier [23, p. 831] to show that for suitable weight functions, which are not of Gevrey type in general, indeed weights which are between two given concrete Gevrey weights, statement (ii) in Theorems 14 and 15 fails if $P$ is not elliptic. Finally, we remark that there is no restriction to assume that the weight $\omega$ is quasianalytic, i.e. satisfies condition $\left(\beta^{\prime}\right)$ and not $(\beta)$, in Theorems 14 and 15. However, in Example 31 the weights are taken to be non-quasianalytic. 


\section{Preliminary results}

In order to prove Theorems 14 and 15 we collect in this section some preliminary results. First of all, we shall prove some properties of the Young conjugate function $\varphi^{*}$ defined in Section 1:

Proposition 21 Let $\omega$ be a subadditive weight function and define, for $j \in \mathbb{N}_{0}$, $\lambda>0$,

$$
a_{j, \lambda}:=\frac{e^{\lambda \varphi^{*}(j / \lambda)}}{j !}
$$

Then the following properties are satisfied:

1. $a_{j, \lambda} \cdot a_{h, \lambda} \leq a_{j+h, \lambda} \quad \forall j, h \in \mathbb{N}_{0}, \lambda>0$;

2. $a_{j, \lambda} \leq a_{j+1, \lambda} \quad \forall j \in \mathbb{N}_{0}, \lambda>0$;

3. $\lambda \mapsto a_{j, \lambda}$ is decreasing for all $j \in \mathbb{N}_{0}$;

4. $a_{j+h, \lambda} \leq a_{j, \lambda / 2} \cdot a_{h, \lambda / 2} \quad \forall j, h \in \mathbb{N}_{0}, \lambda>0$;

5. for every $\rho, \lambda>0$ there exists $\lambda^{\prime}, D_{\rho, \lambda}>0$ such that

$$
\rho^{j} e^{\lambda \varphi^{*}(j / \lambda)} \leq D_{\rho, \lambda} e^{\lambda^{\prime} \varphi^{*}\left(j / \lambda^{\prime}\right)} \quad \forall j \in \mathbb{N}_{0},
$$

with $D_{\rho, \lambda}:=\exp \{\lambda[\log \rho+1]\}$, where $[\log \rho+1]$ is the integer part of $\log \rho+1$

6. for every $j, h, r \in \mathbb{N}_{0}$ with $0 \leq h \leq j$, and for all $\lambda>0$ :

$$
\frac{j !}{h !} a_{j-h, \lambda} \leq \frac{e^{\lambda \varphi^{*}\left(\frac{j+r}{\lambda}\right)}}{e^{\lambda \varphi^{*}\left(\frac{h+r}{\lambda}\right)}}
$$

7. for every $j, h, r \in \mathbb{N}_{0}, \lambda>0$ :

$$
e^{\lambda \varphi^{*}\left(\frac{j}{\lambda}\right)} e^{\lambda \varphi^{*}\left(\frac{r+h}{\lambda}\right)} \leq e^{\frac{\lambda}{2} \varphi^{*}\left(\frac{j+h}{\lambda / 2}\right)} e^{\frac{\lambda}{2} \varphi^{*}\left(\frac{r}{\lambda / 2}\right)} .
$$

8. for every $\lambda>0$ and $q, r \in \mathbb{N}_{0}$ with $q \geq r$ we have that

$$
\frac{e^{\lambda \varphi^{*}\left(\frac{q+1}{\lambda}\right)}}{e^{\lambda \varphi^{*}\left(\frac{q}{\lambda}\right)}} \geq \frac{e^{\lambda \varphi^{*}\left(\frac{r+1}{\lambda}\right)}}{e^{\lambda \varphi^{*}\left(\frac{r}{\lambda}\right)}} .
$$

Proof (1) has been proved in Lemma 3.2.3 of [12].

(2) follows from (1) since $a_{1, \lambda}=e^{\lambda \varphi^{*}(1 / \lambda)} \geq 1$.

(3) follows from the fact that $\varphi^{*}(s) / s$ is increasing (cf. [8]).

(4) follows from the convexity of $\varphi^{*}$ :

$$
\begin{aligned}
a_{j+h, \lambda}=\frac{e^{\lambda \varphi^{*}\left(\frac{j+h}{\lambda}\right)}}{(j+h) !} & \leq \frac{j ! h !}{(j+h) !} \frac{e^{\frac{\lambda}{2} \varphi^{*}\left(\frac{2 j}{\lambda}\right)}}{j !} \frac{e^{\frac{\lambda}{2} \varphi^{*}\left(\frac{2 h}{\lambda}\right)}}{h !} \\
& =\frac{1}{\left(\begin{array}{c}
j+h \\
h
\end{array}\right)} a_{j, \frac{\lambda}{2}} a_{h, \frac{\lambda}{2}} \leq a_{j, \frac{\lambda}{2}} a_{h, \frac{\lambda}{2}} .
\end{aligned}
$$


(5) follows from the next property of [12, Prop. 0.1.5(2)(a)]: for each $y \geq 0$, $n \in \mathbb{N}$, and $\lambda>0$,

$$
\lambda L^{n} \varphi^{*}\left(\frac{y}{\lambda L^{n}}\right)+n y \leq \lambda \varphi^{*}\left(\frac{y}{\lambda}\right)+\lambda \sum_{h=1}^{n} L^{h},
$$

where $L>0$ is such that $\omega($ et $) \leq L(1+\omega(t))$ for all $t \geq 0$ (in our case $\omega$ in increasing and subadditive, so that we can take $L=3$ ).

Indeed, from (2.1) with $y=j L^{n}$ and dividing by $L^{n}$ :

$$
\lambda \varphi^{*}\left(\frac{j}{\lambda}\right)+n j \leq \frac{\lambda}{L^{n}} \varphi^{*}\left(\frac{j}{\lambda / L^{n}}\right)+\lambda \sum_{h=1}^{n} L^{h-n}
$$

and therefore

$$
\rho^{j} e^{\lambda \varphi^{*}\left(\frac{j}{\lambda}\right)} \leq e^{\frac{\lambda}{L^{n}} \varphi^{*}\left(\frac{j}{\lambda / L^{n}}\right)+\lambda n-n j+j \log \rho} .
$$

Choosing $n_{\rho}:=[\log \rho+1] \in \mathbb{N}$ so that $-n_{\rho}+\log \rho \leq 0$, for $\lambda^{\prime}=\lambda / L^{n_{\rho}}$ we thus have that

$$
\rho^{j} e^{\lambda \varphi^{*}\left(\frac{j}{\lambda}\right)} \leq e^{\lambda n_{\rho}} e^{\lambda^{\prime} \varphi^{*}\left(\frac{j}{\lambda^{\prime}}\right)}
$$

so that (5) is proved.

In order to prove (6), let us first remark that

$$
\frac{j !}{h !} a_{j-h, \lambda} \leq \frac{(j+r) !}{(h+r) !} a_{j-h, \lambda}
$$

since $h \leq j$.

From (2.3) we have that

$$
\begin{aligned}
\frac{j !}{h !} a_{j-h, \lambda} & \leq \frac{(j+r) !}{e^{\lambda \varphi^{*}\left(\frac{j+r}{\lambda}\right)}} \cdot \frac{e^{\lambda \varphi^{*}\left(\frac{h+r}{\lambda}\right)}}{(h+r) !} \cdot \frac{e^{\lambda \varphi^{*}\left(\frac{j+r}{\lambda}\right)}}{e^{\lambda \varphi^{*}\left(\frac{h+r}{\lambda}\right)}} a_{j-h, \lambda} \\
& =\frac{a_{h+r, \lambda} a_{j-h, \lambda}}{a_{j+r, \lambda}} \cdot \frac{e^{\lambda \varphi^{*}\left(\frac{j+r}{\lambda}\right)}}{e^{\lambda \varphi^{*}\left(\frac{h+r}{\lambda}\right)}} \leq \frac{e^{\lambda \varphi^{*}\left(\frac{j+r}{\lambda}\right)}}{e^{\lambda \varphi^{*}\left(\frac{h+r}{\lambda}\right)}}
\end{aligned}
$$

by the already proved point (1). Therefore (6) holds true.

Property (7) follows from the convexity of $\varphi^{*}$. Indeed, from (1)

$$
\begin{aligned}
& e^{\lambda \varphi^{*}\left(\frac{j}{\lambda}\right)} e^{\lambda \varphi^{*}\left(\frac{r+h}{\lambda}\right)}=a_{j, \lambda} a_{r+h, \lambda} j !(r+h) ! \\
& \leq a_{j+r+h, \lambda} j !(r+h) !=e^{\lambda \varphi^{*}\left(2 \frac{j+r+h}{2 \lambda}\right)} \frac{j !(r+h) !}{(j+r+h) !} \\
& \leq e^{\frac{\lambda}{2} \varphi^{*}\left(\frac{j+h}{\lambda / 2}\right)+\frac{\lambda}{2} \varphi^{*}\left(\frac{r}{\lambda / 2}\right)} \frac{1}{\left(\begin{array}{c}
j+r+h \\
j
\end{array}\right)} \\
& \leq e^{\frac{\lambda}{2} \varphi^{*}\left(\frac{j+h}{\lambda / 2}\right)} e^{\frac{\lambda}{2} \varphi^{*}\left(\frac{r}{\lambda / 2}\right)} .
\end{aligned}
$$


Let us finally prove (8). We first remark that, by the convexity of $\varphi^{*}$,

$$
2 \varphi^{*}\left(\frac{r+1}{\lambda}\right)=2 \varphi^{*}\left(\frac{r}{2 \lambda}+\frac{r+2}{2 \lambda}\right) \leq \varphi^{*}\left(\frac{r}{\lambda}\right)+\varphi^{*}\left(\frac{r+2}{\lambda}\right)
$$

i.e.

$$
\varphi^{*}\left(\frac{r+1}{\lambda}\right)-\varphi^{*}\left(\frac{r}{\lambda}\right) \leq \varphi^{*}\left(\frac{r+2}{\lambda}\right)-\varphi^{*}\left(\frac{r+1}{\lambda}\right) .
$$

Arguing recursively we get

$$
\varphi^{*}\left(\frac{r+1}{\lambda}\right)-\varphi^{*}\left(\frac{r}{\lambda}\right) \leq \varphi^{*}\left(\frac{q+1}{\lambda}\right)-\varphi^{*}\left(\frac{q}{\lambda}\right)
$$

for every $q \in \mathbb{N}$ with $q \geq r$.

Clearly (2.4) implies (8) and the proof is complete.

For the proof of Theorem 14 we shall follow the ideas of [15], so we define, for a domain $\Omega \subseteq \mathbb{R}^{n}, q \in \mathbb{N}_{0}, \delta>0$ and $f \in C^{\infty}(G)$, with $G$ a relatively compact subdomain of $\Omega$,

$$
\left\|\nabla^{q} f\right\|_{\delta}=\sum_{|\alpha|=q}\left\|D^{\alpha} f\right\|_{L^{2}\left(G_{\delta}\right)}
$$

where

$$
G_{\delta}:=\{x \in G: \operatorname{dist}(x, \partial G)>\delta\}
$$

and $\|\cdot\|_{L^{2}\left(G_{\delta}\right)}=0$ if $G_{\delta}=\emptyset$.

If $P=P(x, D)$ is an elliptic linear partial differential operator of order $m$ with $C^{\infty}$ coefficients, then the following a priori estimates, for $\delta, \sigma>0$ and $0 \leq r \leq m$, have been proved in [14]:

$$
\begin{aligned}
& \left\|\nabla^{m} f\right\|_{\delta+\sigma} \leq C\left(\|P f\|_{\sigma}+\delta^{-m}\|f\|_{\sigma}\right) \\
& \left\|\nabla^{m-r} f\right\|_{\delta+\sigma} \leq C \varepsilon^{r}\left(\left\|\nabla^{m} f\right\|_{\sigma}+\left(\delta^{-m}+\varepsilon^{-m}\right)\|f\|_{\sigma}\right),
\end{aligned}
$$

for arbitrary $\varepsilon>0$, where the constant $C>0$ depends only on the operator $P$ and the set $G$.

Then we define the semi-norm $N^{p m}(u)$ by

$$
N^{p m}(u):=\sup _{0<\delta \leq 1} \delta^{p m}\left\|\nabla^{p m} u\right\|_{\delta} .
$$

The following inequality holds:

Proposition 22 Let $\Omega \subseteq \mathbb{R}^{n}$ be a domain and $P(x, D)$ an elliptic linear partial differential operator of order $m$ with coefficients in $\mathcal{E}_{\{\omega\}}(\Omega)$. For $u \in$ $C^{\infty}(\Omega)$, there exist $k \in \mathbb{N}$ and a positive constant $C_{0}$ such that

$$
N^{p m}(u) \leq C_{0}\left\{N^{(p-1) m}(P u)+\sum_{q=0}^{p-1} \frac{e^{\frac{1}{k} \varphi^{*}(p m k)}}{e^{\frac{1}{k} \varphi^{*}(q m k)}} N^{q m}(u)\right\} .
$$

for every $p \in \mathbb{N}$. 
Proof By definition of the semi-norm $N^{(p+1) m}(u)$ and by $(2.5)$ we have

$$
\begin{aligned}
& N^{(p+1) m}(u)=\sup _{(p+2) \delta \leq 1}((p+2) \delta)^{(p+1) m}\left\|\nabla^{(p+1) m} u\right\|_{(p+2) \delta} \\
& \leq \sup _{(p+2) \delta \leq 1}\left(\frac{p+2}{p}\right)^{(p+1) m}(p \delta)^{(p+1) m} C\left(\left\|P \nabla^{p m} u\right\|_{(p+1) \delta}\right. \\
&\left.\quad+\delta^{-m}\left\|\nabla^{p m} u\right\|_{(p+1) \delta}\right) \\
& \leq 9^{m} C \sup _{(p+2) \delta \leq 1}\left\{(p \delta)^{(p+1) m}\left\|P \nabla^{p m} u\right\|_{(p+1) \delta}\right. \\
&\left.\quad+p^{m}(p \delta)^{p m}\left\|\nabla^{p m} u\right\|_{(p+1) \delta}\right\},
\end{aligned}
$$

since $\left(\frac{p+2}{p}\right)^{p+1} \leq 9$.

We set $P^{[r]}:=\sum_{|\alpha|=r} \sup _{G}\left|D_{x}^{\alpha} P\right|$. Since $\|\cdot\|_{(p+1) \delta} \leq\|\cdot\|_{p \delta}$ and $p^{m}(p m) ! \leq$ $((p+1) m)$ !, from $(2.8)$ and Leibniz' formula we get:

$$
\begin{aligned}
& N^{(p+1) m}(u) \leq 9^{m} C \sup _{(p+2) \delta \leq 1}\left\{(p \delta)^{(p+1) m} \times\right. \\
& \times\left[\left\|\nabla^{p m} P u\right\|_{(p+1) \delta}+\sum_{r=1}^{p m}\left(\begin{array}{c}
p m \\
r
\end{array}\right)\left\|P^{[r]} \nabla^{p m-r} u\right\|_{(p+1) \delta}\right] \\
& \left.+p^{m}(p \delta)^{p m}\left\|\nabla^{p m} u\right\|_{p \delta}\right\} \\
& \leq 9^{m} C \sup _{(p+2) \delta \leq 1}\left\{\left(\frac{p}{p+1}\right)^{p m}[(p+1) \delta]^{p m} \times\right. \\
& \times\left(\frac{p}{p+2}\right)^{m}[(p+2) \delta]^{m}\left\|\nabla^{p m} P u\right\|_{(p+1) \delta} \\
& +(p \delta)^{(p+1) m} \sum_{r=1}^{p m}\left(\begin{array}{c}
p m \\
r
\end{array}\right)\left\|P^{[r]} \nabla^{p m-r} u\right\|_{(p+1) \delta} \\
& \left.+\frac{((p+1) m) !}{(p m) !} N^{p m}(u)\right\} \\
& \leq 9^{m} C\left\{N^{p m}(P u)+\right. \\
& \sup _{(p+2) \delta \leq 1}(p \delta)^{(p+1) m} \sum_{r=1}^{p m}\left(\begin{array}{c}
p m \\
r
\end{array}\right)\left\|P^{[r]} \nabla^{p m-r} u\right\|_{(p+1) \delta} \\
& \left.+\frac{((p+1) m) !}{(p m) !} N^{p m}(u)\right\} \text {. }
\end{aligned}
$$


Taking into account that the coefficients of $P(x, D)$ are in $\mathcal{E}_{\{\omega\}}(\Omega)$, we can write the following estimates, for $(p+2) \delta \leq 1$ and for some $k \in \mathbb{N}$ and $c>0$ :

$$
\begin{gathered}
\sum_{r=1}^{p m}\left(\begin{array}{c}
p m \\
r
\end{array}\right)\left\|P^{[r]} \nabla^{p m-r} u\right\|_{(p+1) \delta} \leq c \sum_{r=1}^{p m}\left(\begin{array}{c}
p m \\
r
\end{array}\right) e^{\frac{1}{k} \varphi^{*}(r k)} \sum_{s=0}^{m}\left\|\nabla^{p m+s-r} u\right\|_{(p+1) \delta} \\
\leq c \sum_{r=1}^{p m} \frac{(p m) !}{(p m-r) !} a_{r, \frac{1}{k}} \sum_{s=0}^{m}\left\|\nabla^{p m+s-r} u\right\|_{(p+1) \delta} .
\end{gathered}
$$

By the change of indexes $r=(p-q) m+t$ we obtain that (cf. also [15])

$$
\begin{aligned}
\sum_{r=1}^{p m}\left(\begin{array}{c}
p m \\
r
\end{array}\right)\left\|P^{[r]} \nabla^{p m-r} u\right\|_{(p+1) \delta} \leq & c(m+1) \sum_{q=1}^{p} \sum_{t=1}^{m} \frac{(p m) !}{(q m-t) !} a_{(p-q) m+t, \frac{1}{k}} \times \\
& \times\left\|\nabla^{(q+1) m-t} u\right\|_{(p+1) \delta} \\
& +c m \sum_{t=1}^{m}(p m) ! a_{p m, \frac{1}{k}}\left\|\nabla^{m-t} u\right\|_{(p+1) \delta} \\
= & c(m+1) \sum_{t=1}^{m} \frac{(p m) !}{(p m-t) !} a_{t, \frac{1}{k}} \times \\
& \times\left\|\nabla^{(p+1) m-t} u\right\|_{(p+1) \delta} \\
& +c(m+1) \sum_{q=1}^{p-1} \sum_{t=1}^{m} \frac{(p m) !}{(q m-t) !} a_{(p-q) m+t, \frac{1}{k}} \times \\
& \times\left\|\nabla^{(q+1) m-t} u\right\|_{(p+1) \delta} \\
& +c m \sum_{t=1}^{m}(p m) ! a_{p m, \frac{1}{k}}\left\|\nabla^{m-t} u\right\|_{(p+1) \delta} . \quad(2.11)
\end{aligned}
$$

From (2.11), by properties (2) and (4) of Proposition 21 we get:

$$
\sum_{r=1}^{p m}\left(\begin{array}{c}
p m \\
r
\end{array}\right)\left\|P^{[r]} \nabla^{p m-r} u\right\|_{(p+1) \delta} \leq S_{1}+S_{2}+S_{3}
$$

with

$$
\begin{aligned}
& S_{1}:=c(m+1) \sum_{t=1}^{m} \frac{(p m) !}{(p m-t) !} a_{m, \frac{1}{k}}\left\|\nabla^{(p+1) m-t} u\right\|_{(p+1) \delta} \\
& S_{2}:=c a_{m, \frac{1}{2 k}}(m+1) \sum_{q=1}^{p-1} \sum_{t=1}^{m} \frac{(p m) !}{(q m-t) !} a_{(p-q) m, \frac{1}{2 k}}\left\|\nabla^{(q+1) m-t} u\right\|_{(p+1) \delta} \\
& S_{3}:=c m \sum_{t=1}^{m}(p m) ! a_{p m, \frac{1}{k}}\left\|\nabla^{m-t} u\right\|_{(p+1) \delta} .
\end{aligned}
$$

By property (3) of Proposition 21 and by (2.6), setting

$$
C_{2}:=9^{m} c C(m+1) a_{m, \frac{1}{2 k}},
$$


we have the estimate

$$
\begin{aligned}
9^{m} C(p \delta)^{(p+1) m} S_{1} \leq & C_{2} \sum_{t=1}^{m} \frac{(p m) !}{(p m-t) !}(p \delta)^{(p+1) m}\left\|\nabla^{(p+1) m-t} u\right\|_{(p+1) \delta} \\
\leq & C_{2} C \sum_{t=1}^{m}(p m)^{t}(p \delta)^{(p+1) m} \varepsilon^{t}\left(\left\|\nabla^{(p+1) m} u\right\|_{p \delta}\right. \\
& \left.\quad+\left(\delta^{-m}+\varepsilon^{-m}\right)\left\|\nabla^{p m} u\right\|_{p \delta}\right) \\
= & C_{2} C \sum_{t=1}^{m}(p m)^{t} \varepsilon^{t}\left\{(p \delta)^{(p+1) m}\left\|\nabla^{(p+1) m} u\right\|_{p \delta}\right. \\
& \left.+\left(p^{m}+(p \delta)^{m} \varepsilon^{-m}\right)(p \delta)^{p m}\left\|\nabla^{p m} u\right\|_{p \delta}\right\},
\end{aligned}
$$

since $(p m) ! \leq(p m-t) !(p m)^{t}$.

Therefore, for $\varepsilon=(p m)^{-1}\left(2 m C C_{2}\right)^{-1 / t}$ and $(p+2) \delta \leq 1$ :

$$
\begin{aligned}
9^{m} C(p \delta)^{(p+1) m} S_{1} \leq & \sum_{t=1}^{m} \frac{1}{2 m}\left\{N^{(p+1) m}(u)\right. \\
& +\left(p^{m}+\left(\frac{p}{p+2}\right)^{m}[(p+2) \delta]^{m} \times\right. \\
& \left.\left.\times(p m)^{m}\left(2 m C C_{2}\right)^{m / t}\right) N^{p m}(u)\right\} \\
\leq & \sum_{t=1}^{m} \frac{1}{2 m}\left\{N^{(p+1) m}(u)\right. \\
\leq & \frac{1}{2} N^{(p+1) m}(u)+C_{3} p^{m} N^{p m}(u) \\
\leq & \frac{1}{2} N^{(p+1) m}(u)+C_{3} \frac{((p+1) m) !}{(p m) !} N^{p m}(u)
\end{aligned}
$$

for some $C_{3}>0$, because of $p^{m}(p m) ! \leq((p+1) m)$ !.

In order to estimate $S_{2}$, let us first prove the following estimate, for $1 \leq$ $q \leq p-1,(p+1) \delta=(q+1) \delta^{\prime}$ and $(p+2) \delta \leq 1$ :

$$
(p \delta)^{(p+1) m} \leq(2 e)^{m}\left(q \delta^{\prime}\right)^{(q+1) m} .
$$


Indeed,

$$
\begin{aligned}
(p \delta)^{(p+1) m} & =\frac{p^{(p+1) m} \delta^{(p+1) m}}{q^{(q+1) m}\left(\frac{p+1}{q+1}\right)^{(q+1) m} \delta^{(q+1) m}} \cdot\left(q \delta^{\prime}\right)^{(q+1) m} \\
& =\left(\frac{p}{p+1} \frac{q+1}{q}\right)^{(q+1) m}(p \delta)^{(p-q) m}\left(q \delta^{\prime}\right)^{(q+1) m} \\
& \leq\left(1+\frac{1}{q}\right)^{q m}\left(1+\frac{1}{q}\right)^{m}\left(\frac{p}{p+2}\right)^{(p-q) m} \times \\
& \leq e^{m} 2^{m}\left(q \delta^{\prime}\right)^{(q+1) m}
\end{aligned}
$$

Therefore (2.14) is proved and, for $1 \leq q \leq p-1,(p+1) \delta=(q+1) \delta^{\prime}$ and $(p+2) \delta \leq 1$ :

$$
\begin{aligned}
& 9^{m} C(p \delta)^{(p+1) m} S_{2} \\
& \leq C_{2} \sum_{q=1}^{p-1} \sum_{t=1}^{m} \frac{(p m) !}{(q m-t) !} a_{(p-q) m, \frac{1}{2 k}}(p \delta)^{(p+1) m}\left\|\nabla^{(q+1) m-t} u\right\|_{(p+1) \delta} \\
& \leq(2 e)^{m} \sum_{q=1}^{p-1} \frac{(p m) !}{(q m) !} a_{(p-q) m, \frac{1}{2 k}} C_{2} \sum_{t=1}^{m} \frac{(q m) !}{(q m-t) !}\left(q \delta^{\prime}\right)^{(q+1) m}\left\|\nabla^{(q+1) m-t} u\right\|_{(q+1) \delta^{\prime}}
\end{aligned}
$$

By (2.13) with $q$ and $\delta^{\prime}$ instead of $p$ and $\delta$ respectively, and because of properties (6) and (2) of Proposition 21 we finally get the following estimate for $S_{2}$ :

$$
\begin{aligned}
& 9^{m} C(p \delta)^{(p+1) m} S_{2} \\
& \leq D \sum_{q=1}^{p-1} \frac{(p m) !}{(q m) !} a_{(p-q) m, \frac{1}{2 k}}\left\{\frac{1}{2} N^{(q+1) m}(u)+C_{3}^{\prime} \frac{((q+1) m) !}{(q m) !} N^{q m}(u)\right\} \\
& \leq D^{\prime} \sum_{q=1}^{p-1}\left(\frac{e^{\frac{1}{2 k} \varphi^{*}(2(p+1) m k)}}{e^{\frac{1}{2 k} \varphi^{*}(2(q+1) m k)}} N^{(q+1) m}+\frac{e^{\frac{1}{2 k} \varphi^{*}(2(p+1) m k)}}{e^{\frac{1}{2 k} \varphi^{*}(2 q m k)}} N^{q m}(u)\right) \\
& \leq 2 D^{\prime} \sum_{q=1}^{p-1} \frac{e^{\frac{1}{2 k} \varphi^{*}(2(p+1) m k)}}{e^{\frac{1}{2 k} \varphi^{*}(2 q m k)}} N^{q m}(u)+D^{\prime} \frac{e^{\frac{1}{2 k} \varphi^{*}(2(p+1) m k)}}{e^{\frac{1}{2 k} \varphi^{*}(2 p m k)}} N^{p m}(u)
\end{aligned}
$$

for some $C_{3}^{\prime}, D, D^{\prime}>0$. 
Let us now estimate $S_{3}$. By (2.6) with $\varepsilon=1$ and because of properties (5), (6) (with $h=0$ ) and (2) of Proposition 21, for $(p+2) \delta \leq 1$ :

$$
\begin{aligned}
& 9^{m} C(p \delta)^{(p+1) m} S_{3} \\
& \leq C_{2} \sum_{t=1}^{m}(p m) ! a_{p m, \frac{1}{k}}(p \delta)^{(p+1) m}\left\|\nabla^{m-t} u\right\|_{(p+1) \delta} \\
& \leq C C_{2} \sum_{t=1}^{m}(p m) !(p \delta)^{p m} a_{p m, \frac{1}{k}}\left((p \delta)^{m}\left\|\nabla^{m} u\right\|_{p \delta}+p^{m}\left(1+\delta^{m}\right)\|u\|_{p \delta}\right) \\
& \leq C C_{2} \sum_{t=1}^{m}(p m) ! a_{p m, \frac{1}{k}}\left(N^{m}(u)+2 p^{m} N^{0}(u)\right) \\
& \leq C C_{2} m(p m) ! a_{p m, \frac{1}{k}} N^{m}(u)+2 C C_{2} m((p+1) m) ! a_{p m, \frac{1}{k}} N^{0}(u) \\
& \leq C C_{2} m \frac{e^{\frac{1}{k} \varphi^{*}((p+1) m k)}}{e^{\frac{1}{k} \varphi^{*}(m k)}} N^{m}(u)+2 C C_{2} m((p+1) m) ! a_{(p+1) m, \frac{1}{k}} N^{0}(u) \\
& \leq \tilde{D} e^{\frac{1}{k} \varphi^{*}((p+1) m k)}\left(N^{m}(u)+N^{0}(u)\right)
\end{aligned}
$$

for some $\tilde{D}>0$.

Substituting $(2.13),(2.15)$ and (2.16) in (2.12) and then in (2.9) and applying (2) of Proposition 21, we finally get:

$N^{(p+1) m}(u) \leq C_{5} N^{p m}(P u)+\frac{1}{2} N^{(p+1) m}(u)+C_{5} \sum_{q=0}^{p} \frac{e^{\frac{1}{k^{\prime}} \varphi^{*}\left((p+1) m k^{\prime}\right)}}{e^{\frac{1}{k^{\prime}} \varphi^{*}\left(q m k^{\prime}\right)}} N^{q m}(u)$,

for some $k^{\prime} \in \mathbb{N}$ and $C_{5}>0$, concluding the proof.

We shall also need, in the following, the next result:

Proposition 23 Let $P(x, D)$ be an elliptic linear partial differential operator of order $m$ with coefficients in $\mathcal{E}_{\{\omega\}}(\Omega)$. For $u \in C^{\infty}(\Omega)$, there are $k \in \mathbb{N}$ and a positive constant $C_{1}>0$ such that

$$
N^{p m}(u) \leq C_{1}^{p} \sum_{q=0}^{p}\left(\begin{array}{l}
p \\
q
\end{array}\right) \frac{e^{\frac{1}{k} \varphi^{*}(p m k)}}{e^{\frac{1}{k} \varphi^{*}(q m k)}} N^{0}\left(P^{q} u\right)
$$

for every $p \in \mathbb{N}_{0}$.

Proof Let us proceed by induction on $p$.

For $p=0$ it's trivial. Let us assume $(2.17)$ to be true for $0,1, \ldots, p-1$ and let us prove it for $p$.

Applying (2.7) for $q \in\{1, \ldots, p-1\}$ instead of $p$, we have that

$$
\begin{aligned}
& N^{m}(u) \leq C_{0}\left\{N^{0}(P u)+e^{\frac{1}{k} \varphi^{*}(m k)} N^{0}(u)\right\} \\
& \vdots \\
& N^{(p-1) m}(u) \leq C_{0}\left\{N^{(p-2) m}(P u)+\sum_{q=0}^{p-2} \frac{e^{\frac{1}{k} \varphi^{*}((p-1) m k)}}{e^{\frac{1}{k} \varphi^{*}(q m k)}} N^{q m}(u)\right\} .
\end{aligned}
$$


Substituting in (2.7) and taking into account (2) of Proposition 21:

$$
\begin{aligned}
& N^{p m}(u) \\
& \leq C_{0}\left\{N^{(p-1) m}(P u)+\frac{e^{\frac{1}{k} \varphi^{*}(p m k)}}{e^{\frac{1}{k} \varphi^{*}((p-1) m k)}} N^{(p-1) m}(u)+\ldots+e^{\frac{1}{k} \varphi^{*}(p m k)} N^{0}(u)\right\} \\
& \leq C_{0}\left\{N^{(p-1) m}(P u)+\frac{e^{\frac{1}{k} \varphi^{*}(p m k)}}{e^{\frac{1}{k} \varphi^{*}((p-1) m k)}} C_{0}\left[N^{(p-2) m}(P u)\right.\right. \\
& \quad \quad \quad \frac{e^{\frac{1}{k} \varphi^{*}((p-1) m k)}}{e^{\frac{1}{k} \varphi^{*}((p-2) m k)}} N^{(p-2) m}(u)+ \\
& \left.\left.\quad \ldots+e^{\frac{1}{k} \varphi^{*}((p-1) m k)} N^{0}(u)\right]+\ldots+e^{\frac{1}{k} \varphi^{*}(p m k)} N^{0}(u)\right\} \\
& \leq C_{0} N^{(p-1) m}(P u)+C_{0}^{2} \frac{e^{\frac{1}{k} \varphi^{*}(p m k)}}{e^{\frac{1}{k} \varphi^{*}((p-1) m k)} N^{(p-2) m}(P u)} \\
& \quad+C_{0}^{2} \frac{e^{\frac{1}{k} \varphi^{*}(p m k)}}{e^{\frac{1}{k} \varphi^{*}((p-2) m k)}} N^{(p-2) m}(u)+\ldots+C_{0}\left(C_{0}+1\right) e^{\frac{1}{k} \varphi^{*}(p m k)} N^{0}(u) \\
& \vdots \\
& \leq \sum_{q=0}^{p-1} \frac{e^{\frac{1}{k} \varphi^{*}(p m k)}}{e^{\frac{1}{k} \varphi^{*}((q+1) m k)}} C_{0}^{p-q} N^{q m}(P u)+\left(C_{0}+1\right)^{p} e^{\frac{1}{k} \varphi^{*}(p m k)} N^{0}(u) \\
& \leq \sum_{q=0}^{p-1} \frac{e^{\frac{1}{k} \varphi^{*}(p m k)}}{e^{\frac{1}{k} \varphi^{*}((q+1) m k)} C_{1}^{p-q} N^{q m}(P u)+C_{1}^{p} e^{\frac{1}{k} \varphi^{*}(p m k)} N^{0}(u)}
\end{aligned}
$$

with $C_{1}:=C_{0}+1$.

Therefore, by the induction assumption and because of property (8) of Proposition 21,

$$
\begin{aligned}
N^{p m}(u) \leq & \sum_{q=0}^{p-1} \frac{e^{\frac{1}{k} \varphi^{*}(p m k)}}{e^{\frac{1}{k} \varphi^{*}((q+1) m k)}} C_{1}^{p-q} C_{1}^{q} \sum_{r=0}^{q}\left(\begin{array}{l}
q \\
r
\end{array}\right) \frac{e^{\frac{1}{k} \varphi^{*}(q m k)}}{e^{\frac{1}{k} \varphi^{*}(r m k)}} N^{0}\left(P^{r} P u\right) \\
& +C_{1}^{p} e^{\frac{1}{k} \varphi^{*}(p m k)} N^{0}(u) \\
\leq & C_{1}^{p} \sum_{r=0}^{p-1} \sum_{q=r}^{p-1} \frac{e^{\frac{1}{k} \varphi^{*}(p m k)}}{e^{\frac{1}{k} \varphi^{*}((r+1) m k)}}\left(\begin{array}{c}
q \\
r
\end{array}\right) N^{0}\left(P^{r+1} u\right) \\
& +C_{1}^{p} e^{\frac{1}{k} \varphi^{*}(p m k)} N^{0}(u) .
\end{aligned}
$$

Let us now remark that $\sum_{q=r}^{p-1}\left(\begin{array}{c}q \\ r\end{array}\right)=\left(\begin{array}{c}p \\ r+1\end{array}\right)$ and hence substituting in (2.18), we finally have:

$$
\begin{aligned}
N^{p m}(u) & \leq C_{1}^{p} \sum_{r=0}^{p-1}\left(\begin{array}{c}
p \\
r+1
\end{array}\right) \frac{e^{\frac{1}{k} \varphi^{*}(p m k)}}{e^{\frac{1}{k} \varphi^{*}((r+1) m k)}} N^{0}\left(P^{r+1} u\right)+C_{1}^{p} e^{\frac{1}{k} \varphi^{*}(p m k)} N^{0}(u) \\
& =C_{1}^{p} \sum_{r^{\prime}=0}^{p}\left(\begin{array}{c}
p \\
r^{\prime}
\end{array}\right) \frac{e^{\frac{1}{k} \varphi^{*}(p m k)}}{e^{\frac{1}{k} \varphi^{*}\left(r^{\prime} m k\right)}} N^{0}\left(P^{r^{\prime}} u\right),
\end{aligned}
$$


so that (2.17) is valid with $C_{1}=1+C_{0}$.

\section{Proof of Theorems 14 and 15}

We can now proceed with the

Proof (Proof of Theorem 14)

Let us first prove that if $P(x, D)$ is elliptic then $\mathcal{E}_{\{\omega\}}^{P}(\Omega) \subseteq \mathcal{E}_{\{\omega\}}(\Omega)$.

Let $u \in C^{\infty}(\Omega)$ satisfy (1) for every $K \subset \subset \Omega$. In particular it satisfies (1) for every relatively compact subdomain $G \subset \Omega$. From Proposition 23, for every fixed $\delta>0$ and for all $p \in \mathbb{N}_{0}$

$$
\begin{aligned}
\left\|\nabla^{p m} u\right\|_{\delta} & \leq \delta^{-p m} N^{p m}(u) \leq \delta^{-p m} C_{1}^{p} \sum_{q=0}^{p}\left(\begin{array}{l}
p \\
q
\end{array}\right) \frac{e^{\frac{1}{k} \varphi^{*}(p m k)}}{e^{\frac{1}{k} \varphi^{*}(q m k)}} N^{0}\left(P^{q} u\right) \\
& \leq \delta^{-p m} C_{1}^{p} \sum_{q=0}^{p}\left(\begin{array}{l}
p \\
q
\end{array}\right) \frac{e^{\frac{1}{k} \varphi^{*}(p m k)}}{e^{\frac{1}{k} \varphi^{*}(q m k)}}\left\|P^{q} u\right\|_{L^{2}(G)} \\
& \leq \delta^{-p m} C_{1}^{p} \sum_{q=0}^{p}\left(\begin{array}{l}
p \\
q
\end{array}\right) \frac{e^{\frac{1}{k} \varphi^{*}(p m k)}}{e^{\frac{1}{k} \varphi^{*}(q m k)}} c e^{\frac{1}{k} \varphi^{*}(q m k)} \\
& \leq c\left(\delta^{-1} C_{1}^{1 / m} 2^{1 / m}\right)^{p m} e^{\frac{1}{k} \varphi^{*}(p m k)} \\
& \leq c D_{\delta} e^{\frac{1}{k^{\prime}} \varphi^{*}\left(p m k^{\prime}\right)}=\tilde{C} e^{\frac{1}{k^{\prime}} \varphi^{*}\left(p m k^{\prime}\right)}
\end{aligned}
$$

for some $k^{\prime} \in \mathbb{N}, D_{\delta}, \tilde{C}>0$, because of (5) of Proposition 21 .

By (2.6) (with $\sigma=\delta, \varepsilon=1, f=\nabla^{p m} u$ ), and by (3.1), for all $1 \leq t \leq m-1$, $t^{\prime}=m-t, q=p m+t$ we have, by the convexity of $\varphi^{*}$ :

$$
\begin{aligned}
\left\|\nabla^{q} u\right\|_{2 \delta} & =\left\|\nabla^{p m+t} u\right\|_{2 \delta}=\left\|\nabla^{m-t^{\prime}} \nabla^{p m} u\right\|_{2 \delta} \\
& \leq C\left(\left\|\nabla^{(p+1) m} u\right\|_{\delta}+\left(\delta^{-m}+1\right)\left\|\nabla^{p m} u\right\|_{\delta}\right) \\
& \leq C \tilde{C}\left[e^{\frac{1}{k^{\prime}} \varphi^{*}\left((p+1) m k^{\prime}\right)}+\left(\delta^{-m}+1\right) e^{\frac{1}{k^{\prime}} \varphi^{*}\left(p m k^{\prime}\right)}\right] \\
& \leq C \tilde{C}\left(2+\delta^{-m}\right) e^{\frac{1}{k^{\prime}} \varphi^{*}\left(((p+1) m+t) k^{\prime}\right)} \\
& \leq C \tilde{C}\left(2+\delta^{-m}\right) e^{\frac{1}{2 k^{\prime}} \varphi^{*}\left(2(p m+t) k^{\prime}\right)} e^{\frac{1}{2 k^{\prime}} \varphi^{*}\left(2 m k^{\prime}\right)} \\
& =C_{\delta} e^{\frac{1}{k^{\prime \prime}} \varphi^{*}\left(q k^{\prime \prime}\right)}
\end{aligned}
$$

for $C_{\delta}=C \tilde{C}\left(2+\delta^{-m}\right) e^{\frac{1}{2 k^{\prime}} \varphi^{*}\left(2 m k^{\prime}\right)}$ and $k^{\prime \prime}=2 k^{\prime}$.

From (3.1) and (3.2), and by Sobolev inequality (cf. [17, Lemma 2.5]), we thus have that $u \in \mathcal{E}_{\{\omega\}}\left(G_{2 \delta}\right)$ for every fixed $\delta>0$ and hence $u \in \mathcal{E}_{\{\omega\}}(\Omega)$.

Let us now show (i). Let $u \in \mathcal{E}_{\{\omega\}}(\Omega)$ and prove by induction on $p$ that there exists $k \in \mathbb{N}$ such that for every $q \in \mathbb{N}_{0}$ there is $C_{q}>0$ such that for every $K \subset \subset \Omega$

$$
\left\|\nabla^{q} P^{p} u\right\|_{L^{2}(K)} \leq C_{q} e^{\frac{1}{k} \varphi^{*}((q+p m) k)} \quad \forall p, q \in \mathbb{N}_{0} .
$$


Indeed, for $p=0$ (3.3) is valid because $u \in \mathcal{E}_{\{\omega\}}(\Omega)$. Let us assume (3.3) to be true for $p$, and all $q \in \mathbb{N}_{0}$, and prove it for $p+1$ :

$$
\begin{aligned}
& \left\|\nabla^{q} P^{p+1} u\right\|_{L^{2}(K)}=\left\|\nabla^{q}\left[P\left(P^{p} u\right)\right]\right\|_{L^{2}(K)}=\sum_{r=0}^{q}\left(\begin{array}{c}
q \\
r
\end{array}\right)\left\|P^{[r]} \nabla^{q-r} P^{p} u\right\|_{L^{2}(K)} \\
& \leq \sum_{r=0}^{q}\left(\begin{array}{c}
q \\
r
\end{array}\right) c e^{\frac{1}{k} \varphi^{*}(r k)} \sum_{s=0}^{m}\left\|\nabla^{q+s-r}\left(P^{p} u\right)\right\|_{L^{2}(K)} \\
& =c \sum_{r=0}^{q} \frac{q !}{(q-r) !} a_{r, \frac{1}{k}}\left\|\nabla^{q+m-r}\left(P^{p} u\right)\right\|_{L^{2}(K)} \\
& \quad+c \sum_{r=0}^{q} \frac{q !}{r !(q-r) !} e^{\frac{1}{k} \varphi^{*}(r k)} \sum_{s=0}^{m-1}\left\|\nabla^{q+s-r}\left(P^{p} u\right)\right\|_{L^{2}(K)}
\end{aligned}
$$

for some $c>0$ since $P(x, D)$ has coefficients in $\mathcal{E}_{\{\omega\}}(\Omega)$.

By property (2) of Proposition 21 we have that, for $0 \leq r \leq q$,

$$
\frac{q !}{(q-r) !} a_{r, \frac{1}{k}} \leq \frac{q !}{(q-r) !} a_{q, \frac{1}{k}} \leq q ! a_{q, \frac{1}{k}}
$$

and hence, substituting in (3.4) and separating the derivatives $\nabla^{\sigma}\left(P^{p} u\right)$ for $\sigma \geq m$ and $0 \leq \sigma \leq m-1$ :

$$
\begin{aligned}
\left\|\nabla^{q} P^{p+1} u\right\|_{L^{2}(K)} \leq & c \sum_{r=0}^{q} \frac{q !}{(q-r) !} a_{r, \frac{1}{k}}\left\|\nabla^{q+m-r}\left(P^{p} u\right)\right\|_{L^{2}(K)} \\
& +m c \sum_{r=0}^{q} \frac{q !}{(q-r) !} a_{r, \frac{1}{k}}\left\|\nabla^{q+m-r}\left(P^{p} u\right)\right\|_{L^{2}(K)} \\
& +m c q ! a_{q, \frac{1}{k}} \sum_{\sigma=0}^{m-1}\left\|\nabla^{\sigma} P^{p} u\right\|_{L^{2}(K)} \\
= & (m+1) c \sum_{r=0}^{q} \frac{q !}{(q-r) !} a_{r, \frac{1}{k}}\left\|\nabla^{q+m-r}\left(P^{p} u\right)\right\|_{L^{2}(K)} \\
& +m c q ! a_{q, \frac{1}{k}} \sum_{\sigma=0}^{m-1}\left\|\nabla^{\sigma}\left(P^{p} u\right)\right\|_{L^{2}(K)} .
\end{aligned}
$$


By the inductive assumption (3.3) and by property (1) of Proposition 21 we have therefore that

$$
\begin{aligned}
\| & \nabla^{q} P^{p+1} u \|_{L^{2}(K)} \leq(m+1) c \sum_{r=0}^{q} \frac{q !}{(q-r) !} a_{r, \frac{1}{k}} C_{q} e^{\frac{1}{k} \varphi^{*}((q+m-r+p m) k)} \\
& +m c q ! a_{q, \frac{1}{k}} \sum_{\sigma=0}^{m-1} C_{q} e^{\frac{1}{k} \varphi^{*}((\sigma+p m) k)} \\
& =(m+1) c C_{q}\left[\sum_{r=0}^{q} \frac{q !}{(q-r) !}(q+(p+1) m-r) ! a_{r, \frac{1}{k}} a_{q+(p+1) m-r, \frac{1}{k}}\right. \\
& \left.+\sum_{\sigma=0}^{m-1} q !(\sigma+p m) ! a_{q, \frac{1}{k}} a_{\sigma+p m, \frac{1}{k}}\right] \\
& \leq(m+1) c C_{q}\left[\sum_{r=0}^{q} \frac{q !}{(q-r) !}(q+(p+1) m-r) ! a_{q+(p+1) m, \frac{1}{k}}\right. \\
& \left.+\sum_{\sigma=0}^{m-1} q !(\sigma+p m) ! a_{q+\sigma+p m, \frac{1}{k}}\right] \\
& =(m+1) c C_{q}\left[\sum_{r=0}^{q} \frac{q !}{(q-r) !} \frac{(q+(p+1) m-r) !}{(q+(p+1) m) !}\right. \\
& \left.+\sum_{\sigma=0}^{m-1} \frac{q !(\sigma+p m) !}{(q+(p+1) m) !}\right] e^{\frac{1}{k} \varphi^{*}((q+(p+1) m) k)} \\
& \leq c C_{q}(m+1)(m+q) e^{\frac{1}{k} \varphi^{*}((q+(p+1) m) k)},
\end{aligned}
$$

since

$$
\frac{q !}{(q-r) !} \frac{(q+(p+1) m-r) !}{(q+(p+1) m) !}=\frac{\left(\begin{array}{c}
q \\
r
\end{array}\right)}{\left(\begin{array}{c}
q+(p+1) m \\
r
\end{array}\right)} \leq 1
$$

and

$$
\frac{q !(\sigma+p m) !}{(q+(p+1) m) !} \leq \frac{1}{\left(\begin{array}{c}
q+(p+1) m \\
q
\end{array}\right)} \leq 1 .
$$

Therefore (3.3) is proved by induction and, in particular, (1) holds true for $q=0$. The proof of Theorem 14 is therefore complete.

Proof (Proof of Theorem 15) The proof of $(i)$ is similar to the Roumieu case, Theorem 14(i), for $C_{q, k}$ and $c_{k}$ instead of $C_{q}$ and $c$.

However, since the constant $C_{1}$ of $(2.17)$ depends on $k$, we cannot deduce formula (3.1) from (5) of Proposition 21. To prove $(i i)$ we first remark that $\mathcal{E}_{\{\omega\}}(\Omega) \subseteq \mathcal{E}_{(\sigma)}(\Omega)$ for $\sigma(t)=o(\omega(t))$ as $t \rightarrow \infty$ by [8, Prop. 4.7]. Therefore by Theorem 14(ii) we have

$$
\mathcal{E}_{(\omega)}^{P}(\Omega) \subseteq \mathcal{E}_{\{\omega\}}^{P}(\Omega) \subseteq \mathcal{E}_{\{\omega\}}(\Omega) \subseteq \mathcal{E}_{(\sigma)}(\Omega)
$$

which concludes the proof in the Beurling case. 
We conclude proving that ellipticity is necessary in Theorems 14(ii) and 15(ii):

Example 31 Let $P(x, D)$ be a linear partial differential operator with real analytic coefficients of order $m$ not elliptic in $\left(x_{0}, \xi_{0}\right) \in \Omega \times \mathbb{R}^{n}$, for a domain $\Omega \subseteq \mathbb{R}^{n}$ and $\left\|\xi_{0}\right\|=1$, i.e.

$$
P_{m}\left(x_{0}, \xi_{0}\right)=0
$$

where $P_{m}$ is the principal part of $P$.

We are going to prove that there exist a function $u$ and a subadditive weight $\omega$, which is not a Gevrey weight in general and is between two given Gevrey weights, and such that $u \in \mathcal{E}_{\{\omega\}}^{P}(\Omega) \backslash \mathcal{E}_{\{\omega\}}(\Omega)$, and that $u \in \mathcal{E}_{(\omega)}^{P}(\Omega) \backslash \mathcal{E}_{(\sigma)}(\Omega)$, for some subadditive weight function $\sigma=o(\omega)$. Consequently, the ellipticity of $P$ is needed for statement (ii) of Theorems 14 and 15.

To construct $\omega$ and the function $u$ we follow [23]: for any fixed $s>1$ we choose $\sigma \in(1, s)$ and $\varepsilon>0$ such that

$$
0<\varepsilon<\frac{m(s-\sigma)}{2 m s-\sigma}<\frac{1}{2} .
$$

Then we take $\delta>0$ so that $B\left(x_{0}, 2 \delta\right) \subset \subset \Omega$ and $\varphi \in \mathcal{E}_{\left(t^{1 / \sigma}\right)}\left(\mathbb{R}^{n}\right)$ with $\operatorname{supp} \varphi \subset$ $B(0,2 \delta)$. For $\eta=\frac{m-\varepsilon}{m s}$ we finally define, as in [23],

$$
u(x):=\int_{1}^{+\infty} \varphi\left(\rho^{\varepsilon}\left(x-x_{0}\right)\right) e^{-\rho^{\eta}} e^{i \rho\left\langle x-x_{0}, \xi_{0}\right\rangle} d \rho .
$$

It was proved in [23] that

$$
\left(D_{\xi_{0}}^{\alpha} u\right)\left(x_{0}\right)=\frac{1}{\eta} \Gamma\left(\frac{\alpha+1}{\eta}\right)+o(1)
$$

where $\Gamma$ is the gamma function, so that $u \notin \mathcal{E}_{\left\{t^{\left.1 / s^{\prime}\right\}}\right.}(U)$ in any neighborhood $U$ of $x_{0}$ for any $s^{\prime}<1 / \eta$ (nor, in particular, for $s^{\prime}=s$ ), but $u \in \mathcal{E}_{\left\{t^{\eta}\right\}}\left(\mathbb{R}^{n}\right)$. Moreover, it was proved in $[23]$ that $u \in \mathcal{E}_{\left\{t^{1 / s}\right\}}^{P}(\Omega)$.

Let us now consider any subadditive weight function $\omega(t)$ such that $\omega(t)=$ $o\left(t^{1 / s}\right)$ and $t^{1 / s^{\prime}}=o(\omega(t))$ for $s^{\prime}>s>1$. For instance, $\omega(t)=t^{1 / s} / \log t$. In general, such a weight exists by [8, Proposition 1.9].

We have that $\mathcal{E}_{(\omega)}(\Omega) \subseteq \mathcal{E}_{\{\omega\}}(\Omega) \subseteq \mathcal{E}_{\left\{t^{1 / s^{\prime}}\right\}}(\Omega)$ and $\mathcal{E}_{\left\{t^{1 / s}\right\}}(\Omega) \subseteq \mathcal{E}_{(\omega)}(\Omega) \subseteq$ $\mathcal{E}_{\{\omega\}}(\Omega)$ by [8, Prop. 4.7]. Analogously $\mathcal{E}_{\left\{t^{1 / s}\right\}}^{P}(\Omega) \subseteq \mathcal{E}_{(\omega)}^{P}(\Omega) \subseteq \mathcal{E}_{\{\omega\}}^{P}(\Omega)$, so that $u \in \mathcal{E}_{\{\omega\}}^{P}(\Omega) \backslash \mathcal{E}_{\{\omega\}}(\Omega)$ and ellipticity is necessary in Theorem 14 (ii).

Moreover, if $\sigma(t):=t^{1 / s^{\prime}}$ we clearly have $u \in \mathcal{E}_{(\omega)}^{P}(\Omega) \backslash \mathcal{E}_{(\sigma)}(\Omega)$. Since $\sigma(t)=$ $o(\omega(t))$ as $t \rightarrow \infty$, this proves that ellipticity is necessary in Theorem 15 (ii). 


\section{References}

1. C. Boiti, D. Jornet, The problem of iterates in some classes of ultradifferentiable functions, "Operator Theory: Advances and Applications", Birkhauser, Basel, 245 (2015), 21-33.

2. C. Boiti, D. Jornet, A characterization of the wave front set defined by the iterates of an operator with constant coefficients, arXiv:1412.4954; submitted for publication.

3. C. Boiti, D. Jornet, J. Juan-Huguet, Wave front set with respect to the iterates of an operator with constant coefficients, Abstr. Appl. Anal. 2014, Article ID 438716 (2014), pp. 1-17, http://dx.doi.org/10.1155/2014/438716

4. P. Bolley, J. Camus, Regularité Gevrey et itérés pour une classe d'opérateurs hypoelliptiques, Comm. Partial Differential Equations 10, n. 6 (1981), 1057-1110.

5. P. Bolley, J. Camus, C. Mattera, Analyticité microlocale et itérés d'operateurs hypoelliptiques, Séminaire Goulaouic-Schwartz, 1978-79, Exp N.13, École Polytech., Palaiseau.

6. P. Bolley, J. Camus, L. Rodino, Hypoellipticité analytique-Gevrey et itérés d'opérateurs, Rend. Sem. Mat. Univers. Politecn. Torino 45, n. 3 (1987), 1-61.

7. J. Bonet, R. Meise, S.N. Melikhov, A comparison of two different ways of define classes of ultradifferentiable functions, Bull. Belg. Math. Soc. Simon Stevin 14 (2007), 425-444.

8. R.W. Braun, R. Meise, B.A. Taylor, Ultradifferentiable functions and Fourier analysis, Result. Math. 17 (1990), 206-237.

9. C. Fernández, A. Galbis, Superposition in classes of ultradifferentiable functions, Publ Res. I. Math. Sci., 42(2) (2006), 399-419.

10. D. Jornet, Operadores Pseudodiferenciales en Clases no Casianalíticas de tipo Beurling, Universidad Politécnica de Valencia, Ph-D Thesis (2003).

11. J. Juan-Huguet, Iterates and Hypoellipticity of Partial Differential Operators on NonQuasianalytic Classes, Integr. Equ. Oper. Theory 68 (2010), 263-286.

12. J. Juan-Huguet, A Paley-Wiener type theorem for generalized non-quasianalytic classes, Studia Math. 208, n.1 (2012), 31-46.

13. H. Komatsu, A characterization of real analytic functions, Proc. Japan Acad. 36 (1960), 90-93.

14. H. Komatsu, On interior regularities of the solutions of principally elliptic systems of linear partial differential equations, J. Fac. Sci., Univ. Tokyo, Sec. 1, 9 (1961), 141-164.

15. H. Komatsu, A Proof of Kotaké and Narasimhan's Theorem, Proc. Japan Acad. 38, n. 9 (1962), 615-618.

16. T. Kotake, M.S. Narasimhan, Regularity theorems for fractional powers of a linear elliptic operator, Bull. Soc. Math. France 90 (1962), 449-471.

17. H. Kumano-Go, Pseudo-differential operators The MIT Press, Cambridge, London, 1982.

18. M. Langenbruch, P-Funktionale und Randwerte zu hypoelliptischen Differentialoperatoren, Math. Ann. 239(1) (1979), 55-74.

19. M. Langenbruch, Fortsetzung von Randwerten zu hypoelliptischen Differentialoperatoren und partielle Differentialgleichungen, J. Reine Angew. Math. 311/312 (1979), 57-79.

20. M. Langenbruch, On the functional dimension of solution spaces of hypoelliptic partial differential operators, Math. Ann. 272 (1985), 217-229.

21. M. Langenbruch, Bases in solution sheaves of systems of partial differential equations, J. reine angew. Math. 373 (1987), 1-36.

22. J.L. Lions, E. Magenes, Problèmes aux limites non homogènes et applications, $\mathbf{3}$, Dunod, Paris (1970).

23. G. Métivier, Propriété des itérés et ellipticité, Comm. Part. Diff. Eq. 3 (9) (1978), 827-876.

24. E. Nelson, Analytic vectors, Ann. of Math. 70 (1959), 572-615.

25. E. Newberger, Z. Zielezny, The growth of hypoelliptic polynomials and Gevrey classes, Proc. Amer. Math. Soc. 39, n. 3 (1973), 547-552.

26. J. Oldrich, Sulla regolarità delle soluzioni delle equazioni lineari ellittiche nelle classi di Beurling, (Italian) Boll. Un. Mat. Ital. (4) 2 (1969), 183-195.

27. H.-J. Petzsche, D. Vogt, Almost analytic extension of ultradifferentiable functions and the boundary values of holomorphic functions, Math. Ann., 267(1) (1984), 17-35. 